\title{
CRECIMIENTO PLANTULAR EN CONDICIONES DE INVERNADERO DE LA ESPECIE FORESTAL Dialium guianense (Aubl.) Sandwith
}

\author{
Seedling growth in greenhouse conditions of the forest species \\ Dialium guianense (Aubl.) Sandwith
}

\author{
Georgina Vargas-Simón ${ }^{1}$, Reinaldo Pire² \& Martha Lázaro Dzul ${ }^{3}$
}

Vargas-Simón, G., Pire, R., \& Lázaro-Dzul, M. (2018). Crecimiento plantular en condiciones de invernadero de la especie forestal Dialium guianense (Aubl.) Sandwith. Colombia Forestal, 21(1), 58-68

Recepción: 9 de febrero de 2017

\section{Resumen}

Dialium guianense es una especie tropical arbórea de bosques perennifolios utilizada por su madera y frutos comestibles. Estos ecosistemas son amenazados, por lo que el objetivo del estudio fue evaluar el crecimiento inicial de la planta con miras a establecer programas de propagación. Las plántulas fueron cultivadas en bolsas de vivero y se empleó un diseño completamente aleatorio con una población inicial de 200 plantas durante 10 meses. El tallo y la raíz alcanzaron longitudes de 32.8 y 28.9 $\mathrm{cm}$, respectivamente. El número de hojas compuestas fue de 12.3 con siete folíolos. La acumulación de biomasa fue de $2.5 \mathrm{~g}$ en el tallo, $1.6 \mathrm{~g}$ en la raíz y 3.7 g en las hojas. La relación vástago/raíz mostró, aproximadamente, cuatro veces mayor biomasa en el vástago. La TRC media fue de $15 \mathrm{mg} \mathrm{g}^{-1} \mathrm{día}^{-1}$. El crecimiento de $D$. guianense resultó lento, indicando que es una especie tardía sucesionalmente.

Palabras clave: biomasa, propagación de plantas, relación vástago/raíz, tasa relativa de crecimiento.
Aprobación: 28 de agosto de 2017

\begin{abstract}
Dialium guianense is used for its wood and fruit production, and is a tropical tree species native to evergreen forests. Given the threat these forests face, the purpose of this work was to evaluate the initial growth of the plant under greenhouse conditions, for aiming in the development of propagation programs. Seedlings of the species were transplanted to nursery bags under a completely randomized design and grown for 10 months with an initial population of 200 plants. At the end of the experiment, the shoot and root reached lengths of 32.8 and $28.9 \mathrm{~cm}$, respectively. The average number of composite leaves was 12.3 each with seven leaflets. The average biomass was $2.5 \mathrm{~g}$ for the shoot, $1.6 \mathrm{~g}$ for roots, and $3.7 \mathrm{~g}$ for leaves, with a shoot/root around four. The average relative growth rate (RGR) was $15 \mathrm{mg} \mathrm{g}-1$ day -1 . These characteristics indicate that $D$. guianense is a late successional species.
\end{abstract}

Keywords: biomass, plant propagation, relative growth rate, shoot/root ratio

\footnotetext{
Universidad Juárez Autónoma de Tabasco. Villahermosa, Tabasco, México. georgina.vargas@ujat.mx. Autor para correspondencia. Universidad Centroccidental Lisandro Alvarado. Barquisimeto, Venezuela. rpire@ucla.edu.ve

Colegio de Postgraduados. Montecillo. Estado de México, México. marodzul@hotmail.com
} 


\section{INTRODUCCIÓN}

D. guianense (Aubl.) Sandwith es una especie arbórea de la familia Fabaceae, subfamilia Caesalpinioideae que tiene una destacada importancia ecológica como especie primaria codominante de los bosques perennifolios de Terminalia amazonia (J.F. Gmel.) Exell, Calophyllum brasiliense Cambess. y Guatteria anomala R.E. Fr. Se encuentra distribuida desde México hasta la Amazonía brasileña Pennington \& Sarukhán (2005), Herrero-Jáuregui et al. (2009) y Wittmann et al. (2013) la ubican en una distribución paleotropical. En la amazonía peruana se encuentra como árbol de sombra del cacao (Vebrova et al., 2014). En México, es una especie restringida a la vertiente del golfo de México. Crece en zonas de topografía ondulada con suelos profundos, lateríticos o derivados de margas calcáreas, arcillosos y de drenaje deficiente. Su amplitud altitudinal va desde el nivel del mar hasta 400 m (Pennington \& Sarukhán, 2005).

El principal producto de D. guianense es la madera, la cual se utiliza para leña y carbón, durmientes, en construcciones pesadas y marinas, en vigas, postes y mangos para herramientas e implementos agrícolas; la pulpa, semejante a la del tamarindo (Tamarindus indica L.), se utiliza para consumirse en fresco y en la elaboración de bebidas refrescantes (Cordero \& Boshier, 2003; Herrero-Jáuregui et al., 2009). Así mismo, los frutos son parte de la dieta de monos (Zárate et al., 2014).

A pesar de la gran importancia de las especies que componen los bosques húmedos, en los últimos años estas áreas han sufrido una considerable reducción debido a las diferentes actividades antropogénicas como el establecimiento de la ganadería, explotación petrolera y sistemas de producción importados, las cuales han traído como consecuencia la disminución de especies como es el caso de $D$. guianense, provocando que esta se remita a relictos de selva o se le encuentre como árboles aislados (Ramírez, 2004).

D. guianense es un árbol de hasta $45 \mathrm{~m}$ de altura y hasta $1.5 \mathrm{~m}$ de diámetro normal; los frutos son vainas de 1 a $2.2 \mathrm{~cm}$ de largo, globosas u ovoides, indehiscentes, con pericarpio frágil, pubescentes de color parduzco y mesocarpio agridulce; contiene una (o dos) semilla reniforme, comprimida, de 5 a 9 mm de largo (Vargas-Simón et al., 2003). Los estudios sobre esta especie son escasos y la mayoría se remiten a su distribución y ecología; solamente existen datos de crecimiento de una especie homóloga nativa de África, Dialium guineense Willd.

Por ser una especie ecológicamente importante, así como valiosa por sus características maderables, el objetivo de este trabajo fue evaluar su crecimiento inicial en condiciones de invernadero, cuyos resultados pueden servir para la implementación de programas de propagación masiva de la especie.

\section{MATERIALES Y MÉTODOS}

\section{Descripción de la zona, colecta de semillas y germinación}

Los frutos maduros se colectaron en HuimanguiIlo, Tabasco, México $\left(17^{\circ} 49^{\prime} 24.70^{\prime \prime}\right.$ latitud norte-93⒉ $24^{\prime} 55.94^{\prime \prime}$ longitud oeste) en una elevación de 34 m de altitud, esta es una zona caracterizada por tener un clima cálido húmedo. La temperatura media es de $27{ }^{\circ} \mathrm{C}$, con valores máximos de $36^{\circ} \mathrm{C}$ en verano y mínimos de $18.5^{\circ} \mathrm{C}$ en el invierno. Las Iluvias son abundantes en el verano, con una precipitación promedio de 2500 mm anuales (Semarnat \& Conafor, 2014).

Las semillas fueron escarificadas mecánicamente por 10 s con lija de papel C-99, 80 SIC de acuerdo con la técnica propuesta por Vargas-Simón et al. (2003) e inmediatamente puestas a germinar en charolas de plástico de 38 celdas, utilizándose arena esterilizada como sustrato.

\section{Trabajo en invernadero}

Una vez que las plántulas tenían 25 días de germinadas y su par de paracotiledones totalmente 
extendidos, se trasplantaron a bolsas de vivero $(30$ $\mathrm{cm}$ de largo x $21 \mathrm{~cm}$ de ancho). El sustrato utilizado consistió en una mezcla de tierra negra, tierra de cacao y germinaza $(1: 1: 1 \mathrm{v} / \mathrm{v})$, previamente desinfectado con Intercaptán $\left(2 \mathrm{gL}^{-1}\right)$. Se dispuso de un total de 200 plantas en igual número de bolsas. Mensualmente se tomaban 10 plantas al azar y se sacrificaban para la evaluación. Esto se repitió durante 10 meses durante los cuales se utilizó un total de 100 plantas. Treinta días después del trasplante (ddt) se iniciaron las evaluaciones del crecimiento vegetativo durante un período de 10 meses a intervalos mensuales, para lo cual se seleccionaron 10 plantas sanas y de tamaño uniforme. El riego se aplicó cada tercer día. El experimento se estableció bajo condiciones de invernadero en la División Académica de Ciencias Biológicas, Universidad Juárez Autónoma de Tabasco (1759'71" latitud norte-92 $58^{\prime} 16^{\prime \prime}$ longitud oeste). Los valores promedios de temperatura y humedad dentro de la estructura registrados fueron $\operatorname{Tmax}=40.6^{\circ} \mathrm{C}$ $\pm 2.7, \mathrm{Tmin}=24.3^{\circ} \mathrm{C} \pm 0.8, \mathrm{HR}=95.9 \% \pm 2.4$. Dentro del invernadero, en días despejados, la radiación fotosintéticamente activa promedio fue de $21.8,117.3$ y $133.0 \mu \mathrm{mol} \mathrm{m} \mathrm{m}^{-2} \mathrm{~s}^{-1}$ a las 9:30, 12:00 y 15:00 horas, respectivamente.

\section{Medición de variables}

Se evaluó la longitud de tallo (LT) y longitud de raíz principal (LR), así como el número de hojas compuestas $(\mathrm{NH})$. Adicionalmente, se midió el diámetro de la base del tallo a los 10 meses después del trasplante; para este momento fue $3.49 \pm$ $0.39 \mathrm{~mm}$. Además, se obtuvo la acumulación de biomasa por órgano, la tasa relativa de crecimiento (TRC) y la relación raíz/vástago. Para obtener la biomasa, cada órgano vegetativo (raíz, tallo y hojas) fue colocado en bolsas de papel estraza y se mantuvieron en la estufa a temperatura constante por $48 \mathrm{~h}$ a $70^{\circ} \mathrm{C}$. Los pesos se obtuvieron mediante una balanza analítica Ohaus (precisión 0.1 g). La tasa relativa de crecimiento (TRC) se obtuvo según la fórmula de Hunt (1982).
Donde:

$$
\mathrm{TCR}=\frac{\ln P S_{2}-\ln P S_{1}}{t_{2}-t_{1}}
$$

InPS 2 : corresponde al logaritmo de peso seco 2. $\mathrm{InPS}_{1}$ : corresponde al logaritmo de peso seco 1. $\mathrm{t}_{2} \mathrm{yt}_{1}$ : corresponden a tiempo 2 y 1 , respectivamente.

\section{Análisis de datos}

El estudio consistió en un ensayo de muestreos con un diseño completamente al azar donde cada muestra estuvo constituida por 10 repeticiones. Los resultados se presentan en forma gráfica incluyendo los valores promedio y su dispersión (barras de error) durante todo el período del ensayo. Adicionalmente, se desarrollaron las ecuaciones de regresión que representan el crecimiento de la biomasa del tallo, raíz y hojas de la planta durante el período total de 300 días después del trasplante. Los cálculos se realizaron utilizando el programa CoStat 6.4 (CoHort Software, Monterey, CA, EE. UU.).

\section{RESULTADOS}

\section{Germinación y crecimiento de tallo, raíz y hojas}

El estudio de crecimiento de las plántulas de $D$. guianense se inició a partir de la germinación de las semillas, la cual ocurrió al tercer día después de la siembra con la salida de la radícula. La emergencia de los cotiledones de almacenamiento, luego de la germinación epígea fanerocotilar, se produjo al séptimo día después de la siembra y los paracotiledones emergieron al décimo.

Al final del periodo de estudio, la LT promedio fue de $32.8 \mathrm{~cm}$, sobrepasando la longitud de la raíz. Esta presentó un aumento gradual a través del periodo de estudio y midió $11.2 \mathrm{~cm}$ en la primera evaluación (30 ddt) hasta alcanzar una longitud final promedio de $28.9 \mathrm{~cm}$ (300 ddt). Cabe resaltar que, a pesar de ser una leguminosa, no se observó nodulación en raíces. El número de hojas 
compuestas promedio fue de 12.3 (figura 1), las cuales se caracterizan por ser imparipinnadas con siete folíolos, ovados con ápice largamente acuminado. El número de folíolos fue variable en esta etapa de crecimiento: a los 30 ddt se formaron los primeros eófilos simples y los trifolíolados, mientras que a los $150 \mathrm{ddt}$ emergieron los metáfilos pentafolíolados, con una filotaxia en espiral.

\section{Acumulación de biomasa}

La acumulación de biomasa por planta al final de la evaluación fue de $3.72 \mathrm{~g}$ en las hojas, $2.53 \mathrm{~g}$ en el tallo y $1.62 \mathrm{~g}$ en las raíces (figura 2). En todos los casos el modelo de regresión explicó casi totalmente la variación en función del tiempo $\left(r^{2}=0.995,0.987\right.$ y 0.990 , respectivamente).

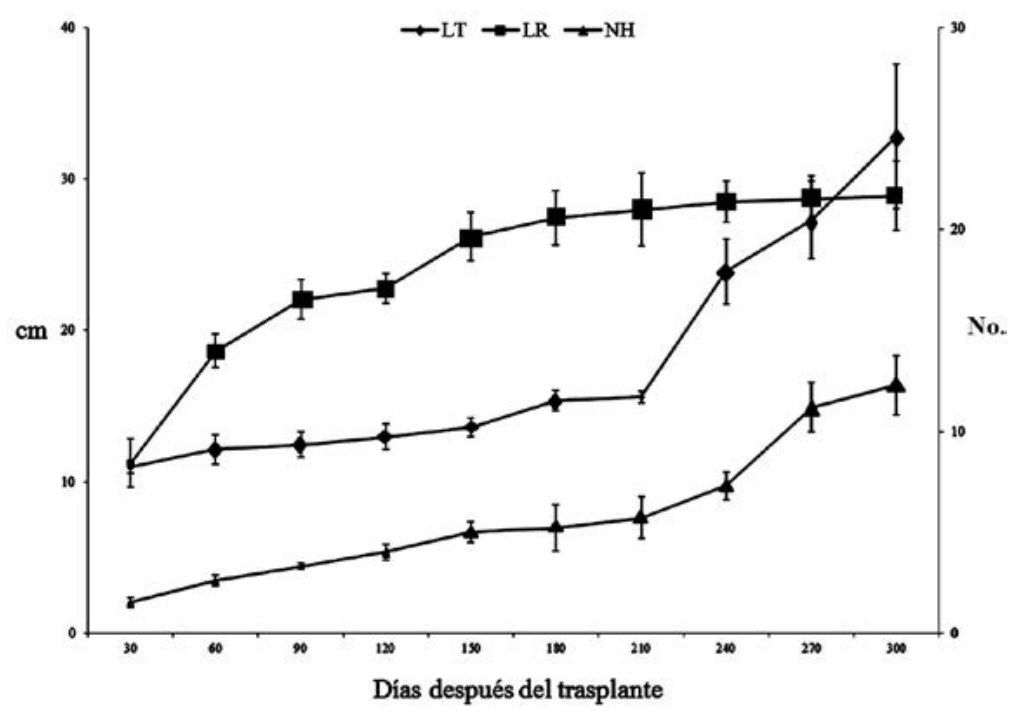

Figura 1. Longitud de tallo (LT), longitud de raíz (LR) y número de hojas $(\mathrm{NH})$ de plántulas de $D$. guianense 300 días después del trasplante. Las barras verticales señalan el error estándar de cada media y cuando no son visibles indica que son más pequeñas que el marcador correspondiente.

\section{$\diamond$ Hojas $\quad$ Tallo $\Delta$ Raíz}

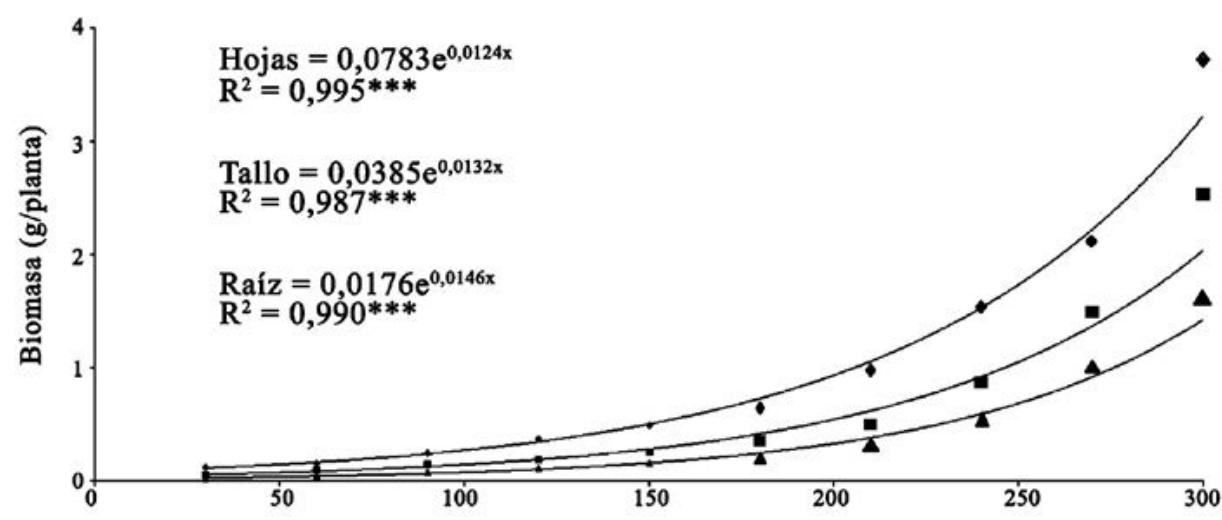

Figura 2. Acumulación de biomasa en hojas, tallo y raíz de $D$. guianense 300 días después del trasplante; $\mathrm{n}=100$. $* * * P \leq 0.001$. 


\section{Tasa relativa de crecimiento $(\mathrm{TRC})$}

La máxima TRC de la planta completa o TRC total se obtuvo a los $60 \mathrm{ddt}$ con un valor promedio de $18.5 \mathrm{mg} \mathrm{g}^{-1} \mathrm{~d}^{-1}$. Al final del experimento fue de $15.5 \mathrm{mg} \mathrm{g}^{-1} \mathrm{~d}^{-1}$ (figura 3).

\section{Relación raíz/vástago}

Referente a la relación raíz/vástago (R/V), se obtuvo un valor promedio final de 0.21 , el cual indica que las plantas de $D$. guianense asignaron en este período de tiempo casi cinco veces más biomasa al vástago que a las raíces (figura 4).

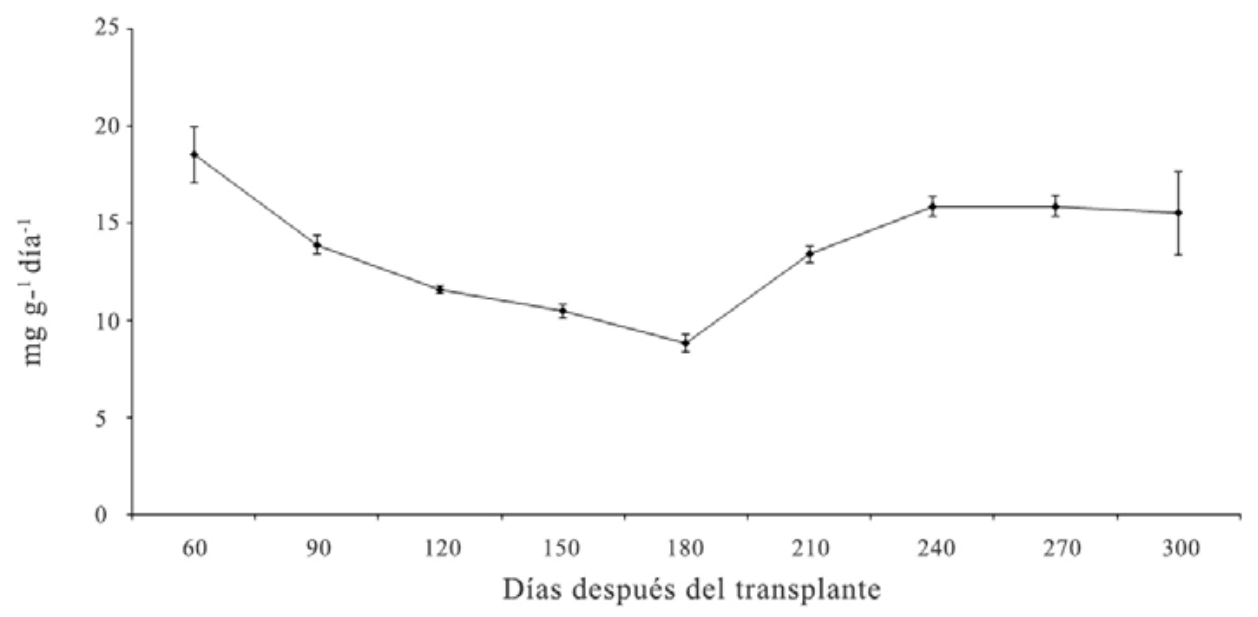

Figura 3. Tasa relativa de crecimiento (TRC) de plantas de $D$. guianense 300 días después del trasplante. Las barras verticales señalan el error estándar de cada media y cuando no son visibles indica que son más pequeñas que el marcador correspondiente.

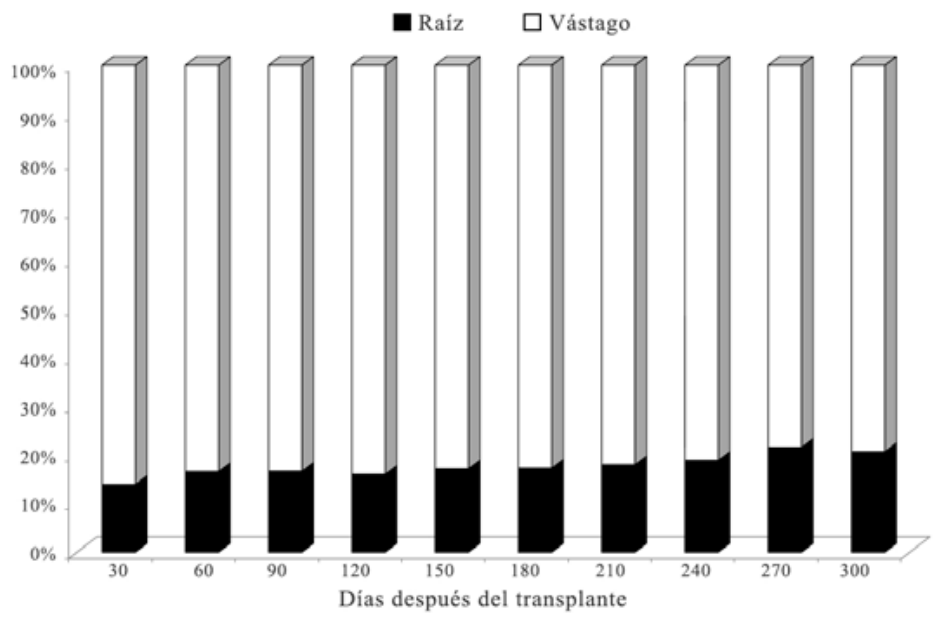

Figura 4. Redistribución de biomasa (\%) en plantas de D. guianense entre la raíz y el vástago 300 días después del trasplante. 


\section{DISCUSIÓN}

\section{Germinación y crecimiento de tallo, raíz y hojas}

En nuestro estudio la germinación se inició a los tres días después de la siembra luego de la escarificación mecánica, lo cual guarda semejanza con el tiempo requerido por la especie Dialium corbisieri Staner, el cual fue de cuatro días (Beaune et al., 2013). Sautu et al. (2006) reportaron que, sin escarificación, el tiempo medio de germinación fue de 32.5 días en condiciones de vivero para $D$. guianense; mientras que, en campo, se ha observado una germinación de solo $12.5 \%$ en 64 días. Lo anterior indica que la especie requiere la utilización de un tratamiento pregerminativo como la escarificación mecánica para promover una rapidez en el proceso. Hartmann et al. (2002) confirman las ventajas de este tratamiento pregerminativo en cuanto a la ganancia en tiempo y homogeneización de la germinación.

La máxima longitud alcanzada por el tallo de la planta al final del estudio $(32.8 \mathrm{~cm})$ indica que D. guianense tiene un crecimiento lento, a pesar de que es una especie codominante en la selva perennifolia (Boege \& Dirzo, 2004). Comparando con especies caesalpinoideas de crecimiento más rápido que Dialium, Santos \& Buckeridge (2004) en Hymenaea courbaril L. reportaron $35 \mathrm{~cm}$ de longitud de tallo a los 50 días de edad; mientras que en Peltophorum dubium (Spreng.) Taub. (Caesalpinoideae) Alves et al. (2013) señalaron $9.3 \mathrm{~cm}$ de tallo a los 14 días. En condiciones de campo, Boege \& Dirzo (2004) registraron un crecimiento promedio del tallo en D. guianense de solo $3.4 \mathrm{~cm}$ en 174 días mientras que Jiménez et al. (2002) señalan que se requieren entre 5 y 7 meses para obtener una altura de $40 \mathrm{~cm}$. Román-Dañobeytia et al. (2012) mostraron que D. guianense en 18 meses después del trasplante alcanza una longitud y un diámetro basal menor a $1 \mathrm{~m}$ y $1.5 \mathrm{~cm}$, respectivamente. Datos similares se registraron en otras especies consideradas tardías sucesionalmente como Brosimum alicastrum Sw. y Ampelocera hottlei (Standl.) Standl.; especies que se caracterizan por ser tolerantes a la sombra y de lento crecimiento (Román-Dañobeytia et al., 2012). En el mismo estudio, las especies pioneras, Muntingia calabura L., Acacia angustissima (Mill.) Kuntze y Ochroma pyramidale (Cav. ex Lam.) Urb., de crecimiento rápido, alcanzan alturas de $2.5 \mathrm{~m}$ y $4 \mathrm{~cm}$ de diámetro basal, particularmente heliófilas (Román-Dañobeytia et al., 2012). Resultados similares de la respuesta al crecimiento entre especies pioneras y tardías sucesionalmente se muestra en Rolim \& Chiarello (2004) y en Román-Dañobeytia et al. (2007).

Investigaciones sobre $D$. guineense han reportado longitudes del tallo de $10.4 \mathrm{~cm}$ a 90 después del trasplante (Olajide et al., 2014) y de $9 \mathrm{~cm}$ a 177 días (Orhue, 1997) para la misma especie africana.

Las plantas se vieron afectadas por una infección fúngica, sin la cual probablemente el crecimiento del tallo pudo haber sido mayor. La longitud de tallo es una de las variables comúnmente usada en viveros para estimar el momento en el que las plantas son adecuadas para ser transportadas a sitios en reforestación (Conafor \& Semarnat, 2005), lo cual se relaciona con su capacidad fotosintética y superficie de transpiración. Las plantas más altas pueden competir ventajosamente con otras especies vegetales en sus cercanías (Quiroz et al., 2009).

La longitud de la raíz presentó un incremento superior a dos veces y media entre la primera (30 ddt) y última medición (300 ddt). Valores similares en esta variable registra Alves et al. (2011) para $P$. dubium, cuya longitud radicular osciló entre 8.9 a $12.5 \mathrm{~cm}$ a los 14 días de edad y Piscidia piscipula L. Sarg. (Papilionoideae), la cual registra una longitud de raíz de $18.7 \mathrm{~cm}$ en promedio a los cuatro meses después del trasplante (Luna-Flores et al., 2012).

Es de destacar que a partir de los $270 \mathrm{ddt}$, las raíces iniciaron la formación de un enroscamiento por la limitación de espacio, por lo que el crecimiento en esta última etapa prácticamente se detuvo y lo cual obligó a decretar la finalización del ensayo en la medición siguiente. Una situación 
similar se presentó en un estudio previo con plantas de Inga jinicuil Schdlt. (Vargas-Simón \& Pire, 2009). La longitud de la raíz es un parámetro que ha mostrado ser un buen predictor de la absorción de agua y nutrientes, aunque su funcionamiento global puede variar entre diferentes raíces y a lo largo de una raíz individual de acuerdo con su edad (Hodge et al., 2009). En las etapas posteriores a la germinación, la raíz tiende a incrementar su tasa de crecimiento, lo cual permite que la plántula se establezca en el sustrato (Azcón-Bieto \& Talón, 2008; Hodge et al., 2009).

Por otra parte, y con relación a la ausencia de nódulos fijadores de nitrógeno, este es un detalle que ya había sido reportado por Orhue (1997) en plantas de $D$. guineense.

Corrrespondiente a la formación de hojas, a mitad del período de evaluación se habían producido cinco hojas por planta, valor comparativo con las siete hojas por planta registrados por Orhue (1997) para $D$. guineense en similar período de tiempo. En el desarrollo plantular de esta especie destacó la heterofilia, es decir, se fueron desarrollando un número diferente de folíolos. El número final corresponde a las hojas adultas según la descripción de Pennington \& Sarukhán (2005). La heterofilia es característica de las leguminosas y otras familias botánicas (Kidner \& Umbreen, 2010; Vozzo, 2010; Alves et al., 2013). Las variantes morfológicas de las hojas están genéticamente programadas, y aunque su función aún no es clara, se menciona que se debe primordialmente a la competencia por luz. Esto sucede sobre todo en las etapas juveniles y, estructuralmente, está relacionado con la filotaxia y la longitud del pecíolo (Kidner \& Umbreen, 2010).

\section{Acumulación de biomasa}

La acumulación de biomasa de 7.87 g por planta durante $300 \mathrm{ddt}$ en $D$. guianense puede considerarse baja si se compara con otras leguminosas tal como Schizolobium parahyba (Vell.) S.F. Blake; una especie de rápido crecimiento que puede alcanzar hasta $9 \mathrm{~g}$ en tallo y cerca de $4 \mathrm{~g}$ en raíces en solo 90 días después del trasplante (De Souza et al., 2012). Datos equivalentes a los registrados en este estudio se observan en Mimosa caesalpiniaefolia Benth y M. hostilis Benth, de 6.1 y 6.2 g planta $^{-1}$, respectivamente, aunque el estudio de Araújo \& Costa (2013) fue de un tiempo menor (90 días). La acumulación de biomasa está relacionada con la cantidad de tejido producido y depende de las actividades de los meristemos, elongación de las células, eficiencia fotosintética y biosíntesis de paredes secundarias, además de los factores ambientales (Demura \& Ye, 2010).

\section{Tasa relativa de crecimiento total (TRC total)}

La máxima TRC obtenida para la planta completa de $D$. guianense fue menor a la TRC de $92 \mathrm{mg} \mathrm{g}^{-1}$ $\mathrm{d}^{-1}$ registrada por Santos \& Buckeridge (2004) para plantas de $H$. courbaril de edad similar, lo cual demuestra el papel preponderante del factor genético sobre esta variable (Villar et al., 2008). La disminución drástica en la TRC total observada en la quinta evaluación (figura 3), es atribuida a la infección fúngica sufrida por las plantas que provocó cierto grado de defoliación y, por consiguiente, una reducción en el crecimiento de toda la planta. Boege \& Dirzo (2004) mencionan que la característica de bajas tasas de crecimiento en las plantas puede estar determinada por una respuesta adaptativa a una posible baja disponibilidad de recursos; pero también porque es un árbol cuya madera tiene una alta densidad, $1.02 \mathrm{~g} \mathrm{~cm}^{-3}$ (Flores-Vindas \& Obando-Vargas, 2003). King et al. (2006), muestran una relación indirectamente proporcional entre la tasa de crecimiento relativa y la densidad de madera en algunas especies tropicales, entre ellas Dialium indum L. cuya tasa promedio es de $2.27 \mathrm{~mm}^{2}$ ño ${ }^{-1} \mathrm{y}$ una densidad de $0.82 \mathrm{~g} \mathrm{~cm}^{-3}$, se requiere un gasto energético mayor para construir un tejido consistente. Esta concordancia también es corroborada por Muller-Landau (2004) quien evaluó árboles tropicales en Costa Rica, Panamá, Perú y Brasil; de la misma forma, Poorter et al. (2010) afirman que 
el costo de construcción de madera de baja densidad es menor, lo que permite que los tallos de los árboles crezcan más rápidamente.

\section{Relación raíz/vástago}

El valor obtenido para la relación raíz/vástago en este estudio es concordante con lo reportado por Baskin et al. (1999) en plantas de Senna obtusifolia (L.) H.S. Irwin \& Barneby (Papilionoideae), las cuales también asignaron una mayor proporción de asimilados al vástago más que a las raíces (relación de 0.073), pero difiere en lo reportado por los mismos autores para plantas de la especie $S$. marilandica (L.) Link, cuyas plantas asignaron una mayor cantidad a las raíces (relación de 0.859). En este último caso, se interpreta que lo primordial es disponer de un mejor anclaje y mayor capacidad de absorción de agua y nutrientes cuando los recursos del suelo son limitantes (Villar et al., 2008).

Al respecto, Mou et al. (2013) señalan que los patrones de asignación de recursos varían dependiendo de la identidad de las especies y edad de la planta. La influencia de estos factores puede observarse más claramente en este estudio, ya que al inicio del periodo de crecimiento las plantas asignaron una mayor cantidad de recursos particularmente hacia el vástago. Lo anterior posiblemente se deba al hecho de que $D$. guianense es una especie de germinación epígea, con cotiledones activos, que junto con las hojas realizarían un trabajo intenso fotosintético para darle a la plántula mayor cantidad de asimilados y promover su crecimiento (Villar et al., 2008); el mismo comportamiento se observó en $H$. courbaril, cuya relación fue de 0.517; esta es una especie intolerante a la sombra y con buena elasticidad ecológica (Guzmán et al., 2013). Al final del periodo de estudio las plantas empezaron a dirigir relativamente más asimilados hacia las raíces (20.6\%); esto implicará una mayor superficie de absorción y un mejor crecimiento para las etapas posteriores. Mou et al. (2013) mencionan que cuando un ambiente de suelo es estable, se promueve el crecimiento de raíces finas porque ellas son más eficientes en la absorción de agua y nutrientes y la planta evita elevar los costos fisiológicos de producción de raíces grandes en un ambiente impredecible.

En condiciones de campo, las plántulas de $D$. guianense tienen una supervivencia muy baja cuando se siembran directamente (Román-Dañobeytia et al., 2012), por lo que es imprescindible para su propagación mantenerlas en invernadero suficiente tiempo hasta que la planta tenga la longitud de tallo y número de hojas necesarios para adecuarse al nuevo ambiente, tal como lo mencionan Meli et al. (2013), quienes recomiendan esta especie para programas de restauración activa.

\section{CONCLUSIONES}

D. guianense presentó una germinación epígea fanerocotilar con cotiledones de almacenamiento y un crecimiento lento, comparativamente con otras especies arbóreas de la misma familia, al menos hasta los 10 meses de cultivo en condiciones de vivero. Durante ese tiempo, la planta acumuló 7.87 g de biomasa y alcanzó $32.8 \mathrm{~cm}$ de altura. La máxima tasa relativa de crecimiento se produjo a los 60 días con un valor de $18.5 \mathrm{mg} \mathrm{g}^{-1} \mathrm{~d}^{-1}$. Estos indicadores pueden servir de antecedentes para su posterior propagación y definen a esta especie como tardía sucesionalmente. No obstante, el alto valor económico de su madera permite recomendarla en programas de conservación y restauración.

\section{CONFLICTO DE INTERESES}

Los autores declaran no tener conflicto de intereses.

\section{CONTRIBUCIÓN POR AUTOR}

El autor único es responsable de la obra en todos los aspectos que condujeron a la elaboración de su publicación. 


\section{REFERENCIAS BIBLIOGRÁFICAS}

Alves, E.U., de Andrade, L.A., Bruno, R. de L.A., Vieira R.M., \& Cardoso, E. de A. (2011). Emergência e crescimento inicial de plântulas de Peltophorum dubium (Spreng.) Taubert sob diferentes substratos. Revista Ciência Agronômica, 42, 439-447. DOI: https://doi.org/10.1590/S1806-66902011000200025

Alves, L.M.C.J., Lima, P.B., Lima, L.F., \& Zickel, C.S. (2013). Descrição morfológica para identificação das plántulas de nove espécies lenhosas de uma floresta de restinga. Biota Neotropical, 3, 374-383. DOI: https://doi.org/10.1590/ S1676-06032013000300036

Araújo, I.C.S., \& Costa, M.C.G. (2013). Biomass and nutrient accumulation pattern of leguminous tree seedlings grown on mine tailings amended with organic waste. Ecological Engineering, 60, 254-260. DOI: https://doi.org/10.1016/j.ecoleng.2013.07.016

Azcón-Bieto, J., \& Talón, M. (2008). Fundamentos de fisiología vegetal. Madrid: McGraw-Hill-Interamericana. $522 \mathrm{p}$.

Baskin, M.J., Nan, X., \& Baskin, C.C. (1999). A comparative study of the seedling-juvenile and flowering stages of the life cycle in an annual and perennial species of Senna (Leguminosae; Caesalpinioideae). American Midland Naturalist, 141, 381-390. DOI: https://doi.org/10.1674/0003-0031(1999)141[038 1:ACSOTS]2.0.CO;2

Beaune, D., Bollache, L., Muganza, M. D., Bretagnolle, F., Hohmann, G., \& Fruth, B. (2013). Artificial germination activation of Dialium corbisieri by imitation of ecological process. Journal of Sustainable Forestry, 32, 565-575. DOI: https://doi.org/10.108 0/10549811.2013.780199

Boege, K., \& Dirzo, R. (2004). Intraspecific variation in growth, defense and herbivory in Dialium guianense (Caesalpiniaceae) mediated by edaphic heterogeneity. Plant Ecology, 175, 59-69. DOI: https:// doi.org/10.1023/B:VEGE.0000048092.82296.9a

Comisión Nacional Forestal \& Secretaría de Medio Ambiente y Recursos Naturales (Conafor \& Semarnat). (2005). Manual práctico para producción de planta. Programa Nacional de Reforestación,
Coordinación de Conservación y Restauración. Zapopan: Comisión Nacional Forestal, Secretaría de Medio Ambiente y Recursos Naturales. 197 p.

Cordero, J., \& Boshier, D.H. (2003). Árboles de centroamérica, un manual para extensionistas. Costa Rica: Oxford Forestry Institute, Centro Agronómico Tropical de Investigación y Enseñanza (Catie), CD-ROM.

De Souza, T.V., Voltolini, C.H., Santos, M., \& Paulilo, M.T.S. (2012). Water absorption and dormancy-breaking requirements of physically dormant seeds of Schizolobium parahyba (Fabaceae-Caesalpinioideae). Seed Science Research, 22, 169-176. https://doi.org/10.1017/S0960258512000013

Demura, T., \& Ye, Z.H. (2010). Regulation of plant biomass production. Current Opinion in Plant Biology, 13, 298-303. DOI: https://doi.org/10.1016/j. pbi.2010.03.002

Flores-Vindas, E., \& Obando-Vargas, G. (2003). Árboles del trópico húmedo Importancia socioeconómica (pp. 317-322). Cartago: Editorial Tecnológica de Costa Rica.

Guzmán, Q., Antonio, J., Cordero, S., \& Roberto, A. (2013). Growth and photosynthetic performance of five tree seedlings species in response to natural light regimes from the Central Pacific of Costa Rica. Revista de Biología Tropical, 61, 1433-1444.

Hartmann, H.T., Kester, D.E., \& Geneve, R.L. (2002). Plant Propagation. Principles and Practices. New Jersey: Prentice-Hall. 880 p.

Herrero-Jáuregui, C., García-Fernández, C., Sist, P.L., \& Casado, M.A. (2009). Conflict of use for multi-purpose tree species in the state of Pará, eastern Amazonia, Brazil. Biodiversity and Conservation, 18, 1019-1044. DOI: https://doi.org/10.1007/ s10531-008-9456-7

Hodge, A., Berta, G., Doussan, C., Merchan, F., \& Crespi, M. (2009). Plant root growth, architecture and function. Plant Soil, 321, 153-187. DOI: https://doi. org/10.1007/s11104-009-9929-9

Hunt, R. (1982). Plant growth curves: The functional approach to plant growth analysis. Baltimore: University Park Press. 248 p.

Jiménez, M.Q., Rojas, F.E.R., Rojas, V.C., \& Rodríguez, L.S. (2002). Árboles maderables de Costa Rica: 
ecología y silvicultura. Santo Domingo de Heredia: Instituto Nacional de Biodiversidad. 361 p.

Kidner, A.C., \& Umbreen, S. (2010). Why is leaf shape so variable? International Journal of Plant Developmental Biology, 3, 64-75.

King, D.A., Davies, S.J., Tan, S., \& Noor, N. S. (2006). The role of wood density and stem support costs in the growth and mortality of tropical trees. Journal of Ecology, 94, 670-680. DOI: https://doi. org/10.1111/j.1365-2745.2006.01112.x

Luna-Flores, W., Estrada-Medina, H., Jiménez-Osornio, J.J.M., \& Pinzón-López, L.L. (2012). Efecto del estrés hídrico sobre el crecimiento y eficiencia del uso del agua en plántulas de tres especies arbóreas caducifolias. Terra Latinoamericana, 30, 343-353.

Meli, P., Martínez-Ramos, M., \& Rey-Benayas, J.M. (2013). Selecting species for passive and active riparian restoration in Southern Mexico. Restoration Ecology, 21, 163-165. DOI: https://doi. org/10.1111/j.1526-100X.2012.00934.x

Mou, P., Jones, R.H., Tan, Z., Bao, Z., \& Chen, H. (2013). Morphological and physiological plasticity of plant roots when nutrients are both spatially and temporally heterogeneous. Plant Soil, 354, 373-384. DOI: https://doi.org/10.1007/s11104-012-1336-y

Muller-Landau, H.C. (2004). Interspecific and inter-site variation in wood specific gravity of tropical trees. Biotropica, 35, 20-32. DOI: https://doi. org/10.1646/02119

Olajide, O., Oyedeji, A.A., Tom, G.S., \& Kayode, J. (2014). Seed germination and effects of three watering regimes on the growth of Dialium guineense (Wild) seedlings. American Journal of Plant Sciences, 5, 3049-3059. DOI: https://doi.org/10.4236/ ajps.2014.520321

Orhue, R.E. (1997). Compound fertilizer effects on growth of Dialium guineense seedlings. Forest, Farm and Community Tree Research Reports, 2, 38-40.

Pennington, T.D., \& Sarukhán, J. (2005). Árboles tropicales de México. (pp. 232-233). México, D.F.: Universidad Nacional Autónoma de México, Fondo de Cultura Económica.

Poorter, L., McDonald, I., Alarcón, A., Fichtler, E., Licona, J.C., Peña-Claros, M., Sterck, F., Villegas, Z., \&
Sass-Klaassen, U. (2010). The importance of wood traits and hydraulic conductance for the performance and life history strategies of 42 rainforest tree species. New Phytologist, 185, 481-492. DOI: https://doi.org/10.1111/j.1469-8137.2009.03092.x

Quiroz, M.I., García, E.R., González, M.O., Chung, P.G.P., \& Soto, H.G. (2009). Vivero forestal. Producción de plantas nativas a raíz cubierta. Concepción, Chile: Centro Tecnológico de la planta forestal, Gobierno de Chile. 128 p.

Ramírez, M.L. (2004). Distribución y áreas con potencial productivo de nueve especies arbóreas de la selva alta perennifolia en México. (Tesis de Maestría en Ciencias Biológicas). México, D.F.: Universidad Nacional Autónoma de México. 112 p.

Rolim, S.G., \& Chiarello, A.G. (2004). Slow death of Atlantic forest trees in cocoa agroforestry in southeastern Brazil. Biodiversity and Conservation, 13, 2679-2694. DOI: https://doi.org/10.1007/ s10531-004-2142-5

Román-Dañobeytia, F., Levy-Tacher, S., Perales-Rivera, H., Ramírez-Marcial, N., Douterlungne, D., \& López-Mendoza, S. (2007). Establecimiento de seis especies arbóreas nativas en un pastizal degradado en la selva lacandona, Chiapas, México. Ecología Aplicada, 6, 1-8. DOI: https://doi.org/10.21704/ rea.v6i1-2.335

Román-Dañobeytia, F.J., Levy-Tacher, S.I., Aronson, J., Rodrigues, R.R., \& Castellanos-Albores, J. (2012). Testing the performance of fourteen native tropical tree species in two abandoned pastures of the Lacandon rainforest region of Chiapas, Mexico. Restoration Ecology, 20, 378-386. DOI: https://doi. org/10.1111/j.1526-100X.2011.00779.x

Santos, P.H., \& Buckeridge, M.S. (2004). The role of the storage carbon of cotyledons en the establishment of seedlings of Hymenaea courbaril under different light conditions. Annals of Botany, 94, 819-830. DOI: https://doi.org/10.1093/aob/mch209

Sautu, A., Baskin, J.M., Baskin, C.C., \& Condit, R. (2006). Studies on the seed biology of 100 native species of trees in a seasonal moist tropical forest, Panama, Central America. Forest Ecology and 
Management, 234, 245-263. DOI: https://doi.org/10.1016/j.foreco.2006.07.006

Secretaría de Medio Ambiente y Recursos Naturales (Semarnat), \& Comisión Nacional Forestal (Conafor) (2014). Inventario estatal forestal y de suelos, Guadalajara. Guadalajara: Semarnat y Conafor. $124 \mathrm{p}$.

Vargas-Simón, G., Molina-Martínez, R.F., \& Moguel-Ordoñez, E.J. (2003). Caracterización del fruto, semilla y estudios de germinación del guapaque (Dialium guianense (Aubl) Sandwith). Universidad y Ciencia, 19, 77-83.

Vargas-Simón, G., \& Pire, R. (2009). Efecto de la longitud radicular al momento del transplante sobre el crecimiento vegetativo del cuijinicuil (Inga jinicuil Schtdl, \& Cham. ex. G. Don) en invernadero. Agrociencia, 43, 603-608.

Vebrova, H., Lojka, B., Husband, T.P., Zans, M.E.C., Van Damme, P., Rollo, A., \& Kalousova, M. (2014). Tree diversity in cacao agroforests in San Alejandro, Peruvian Amazon. Agroforestry Systems, 88, 1101-1115. DOI: https://doi.org/10.1007/ s10457-013-9654-5
Villar, R., Ruiz-Robleto, J., Quero, J.L., Poorter, H., Valladares, F., \& Marañón, T. (2008). Tasas de crecimiento en especies leñosas: aspectos funcionales e implicaciones ecológicas. En: F. Valladares Ecología del bosque mediterráneo en un mundo cambiante (pp. 193-230). Madrid: Ministerio de Medio Ambiente. EGRAF, S. A.

Vozzo, J.A. (2001). Tropical tree seed manual. Washington D.C.: USDA Forest Service, Agriculture Handbook. $721 \mathrm{p}$.

Wittmann, F., Householder, E., Piedade, M.T., de Assis, R.L., Schöngart, J., Parolin, P., \& Junk, W.J. (2013). Habitat specifity, endemism and the neotropical distribution of Amazonian white-water floodplain trees. Ecography, 36, 690-707. DOI: https://doi. org/10.1111/j.1600-0587.2012.07723.x

Zárate, D. A., Andresen, E., Estrada, A., \& Serio-Silva, J. C. (2014). Black howler monkey (Alouatta pigra) activity, foraging and seed dispersal patterns in shaded cocoa plantations versus rainforest in southern Mexico. American Journal of Primatology, 76, 890899. DOI: https://doi.org/10.1002/ajp.22276 